\title{
Radiotherapy and immune checkpoint blockades: a snapshot in 2016
}

\author{
Taeryool Koo, MD', In Ah Kim, MD, PhD² \\ 'Department of Radiation Oncology, Hallym University Chuncheon Sacred Heart Hospital, Chuncheon; \\ ${ }^{2}$ Department of Radiation Oncology, Seoul National University College of Medicine, Seoul, Korea
}

Immune checkpoint blockades including monoclonal antibodies (mAbs) of cytotoxic T-lymphocyte antigen-4 (CTLA-4), programmed death-1 (PD-1), and programmed death-ligand 1 (PD-L1) have been emerged as a promising anticancer therapy. Several immune checkpoint blockades have been approved by US Food and Drug Administration (FDA), and have shown notable success in clinical trials for patients with advanced melanoma and non-small cell lung cancer. Radiotherapy is a promising combination partner of immune checkpoint blockades due to its potent pro-immune effect. This review will cover the current issue and the future perspectives for combined with radiotherapy and immune checkpoint blockades based upon the available preclinical and clinical data.

Keywords: Radiotherapy, Immune checkpoint blockades, Cytotoxic T-lymphocyte antigen-4, Programmed cell death 1 receptor

\section{Introduction}

Radiotherapy (RT) is a main modality for anticancer treatment. Radiation directly ionizes atoms of the cancer cells and makes excited electrons. These secondary electrons directly damage the DNA of cancer cells. The electrons also make free radicals by interacting with water in the tissue, which can damage the DNA of cancer cells. This is the indirect action of radiation. Incomplete repair of these damaged DNA causes several modes of cell death, such as apoptosis, autophagy, or senescence. Radiosensitivity varies according to cells, tissues, and organs. In particular, lymphocytes show high radiosensitivity even in a low dose, so RT has been used as an immunosuppressive therapy, such as total body irradiation for conditioning prior to bone marrow transplantation. Also, RT can activate immunosuppressive transforming growth factor- $\beta$ (TGF- $\beta$ ) and tumor promoting macrophage [1]. However, RT can induce immunologic cell death providing tumor specific peptides presented by major histocompatibility complex (MHC) class I of antigen-presenting cells (APCs) and recognized by cytotoxic T cells [2]. Furthermore, RT also can activate APCs possessing anti-tumor immunity [3]. These T cells infiltrate to the tumor site, secrete interferon $\gamma$ (IFN- $\gamma$ ), and kill tumor cells [4]. Specific combination of signals could be released either, which stimulate tumor-specific cytotoxic T lymphocytes even in the distant sites, so-called "abscopal effect." The systemic effects induced by local RT have been reported in patients with several types of solid tumors, such as melanoma, renal cell carcinoma, and lung adenocarcinoma [5] (Table 1).

Received 14 November 2016, Revised 12 December 2016, Accepted 13 December 2016.

Correspondence: In Ah Kim, MD, PhD, Department of Radiation Oncology, Seoul National University Bundang Hospital, 82 Gumi-ro 173beon-gil, Bundang-gu, Seongnam 13620, Korea. Tel: +82-31-787-7651, Fax: +82-31-787-4019, E-mail: inah228@ snu.ac.kr

(c) This is an Open Access article distributed under the terms of the Creative Commons Attribution Non-Commercial License (http://creativecommons.org/ licenses/by-nc/4.0/) which permits unrestricted non-commercial use, distribution, and reproduction in any medium, provided the original work is properly cited.

www.e-roj.org 
ROJ Radiation Oncology Journal

\section{Immune Checkpoint Blockades}

Immune checkpoint blockades have been showing remarkable progress in the field of immunotherapy, regulating key immunosuppressive pathways of cancer cells. Targets of checkpoint blockades are cytotoxic T-lymphocyte antigen-4 (CTLA-4) and programmed death-1 (PD-1), crucial molecules for peripheral CD8+ T cell tolerance induced by APC. CTLA-4, a trans-membrane protein receptor which expressed in T cells, affects priming phase of immune response. It is transported to the surface when $\mathrm{T}$ cell receptor (TCR) recognizes an antigenic peptide in association with MHC of APC. For the complete $T$ cell stimulation, $C D 28$ receptor of $T$ cell and $B 7$ ligand of APC are needed to be bound for a co-stimulatory pathway [6]. CTLA-4 has higher affinity, thus inhibits proliferation of T cells by outcompeting CD28 receptor for ligand binding. CTLA4 mediated $T$ cell immune tolerance also can be achieved by production of cytokines such as TGF- $\beta$ in regulatory T cells [7]. Another key inhibitory receptor PD-1 is on surface of T cell and $\mathrm{B}$ cell, and binds to programmed death-ligands 1 and 2 (PD-L1 and PD-L2). PD-L1 is widely expressed on hematopoietic and non-hematopoietic cells. The main role of PD-1/PD-L1 system is to limit the response of effecter $T$ cell and the immunemediated damage of tissues. PD-L1 is also expressed on various types of solid tumors and hematologic malignancies. Tumor cells with PD-L1 expression can escape from T cell related immune reaction, and this adaptive resistance is regulated by cytokines, such as tumor necrosis factor- $\alpha$ (TNF- $\alpha$ ) and IFN- $\gamma[8]$.

In clinical trials using the checkpoint blockades, anti-CTLA-4 and anti-PD-1 monoclonal antibodies (mAbs), improved survival outcomes were reported for patients with advanced solid tumors, in particular melanoma and non-small cell lung cancer (NSCLC). Ipilimumab, an anti-CTLA-4 mAb, was administered for metastatic melanoma. Response rate was $11 \%$ to $15.2 \%$ and median overall survival (OS) time was 10.1 to 11.2 months. Adverse effect rate was higher, grade 3 to 4 events occurred in $45 \%$ to $60 \%$ of patients. Of these, immunerelated adverse effects were $10 \%$ to $41.7 \%$. Common side effects were skin reaction, diarrhea, and increased liver enzyme $[9,10]$. Interestingly, survival benefit was lasted for long time, a plateau was found after 3 -year, and OS rate was 18.2\% at 5-year [11]. Anti-PD-1 mAbs (nivolumab and pembrolizumab), comparing with ipilimumab, were reported to prolong survival and response rate, while decrease adverse effect in metastatic melanoma patients $[12,13]$. In the trial using pembrolizumab (vs. ipilimumab), 1-year OS rate was 68.4\%-74.1\% (vs. 58.2\%),
Immune checkpoint blockade combined with radiotherapy

response rate was $32.9 \%-33.7 \%$ (vs. $11.9 \%$ ), and grade 3 to 5 adverse effect rate was $10.1 \%$ to $13.3 \%$ (vs. 19.9\%) [13].

In the patients with chemo-refractory NSCLC, nivolumab showed better treatment outcomes than docetaxel. Median OS was 9.2-12.2 vs. 6.0-9.4 months and response rate was 19\%-20\% vs. 9\%-12\%. Grade 3 to 4 adverse events were less in nivolumab group, $7 \%-10 \%$ vs. $54 \%-55 \%$. Of these patients having response to nivolumab, response duration time was very long, median time was 17.2 months or unreached $[14,15]$. Pembrolizumab also compared with docetaxel in PDL1-positive NSCLC patients who had history of treatment. Median OS was 10.4-12.7 months vs. 8.5 months, and response rate was $18.0 \%-18.5 \%$ vs. 9.3\%. Patients with PDL1 positive in 50\% or more tumor cells had more improved outcomes, median OS was 14.9-17.3 months vs. 8.2 months, and response rate was $29.1 \%-30.2 \%$ vs. $7.9 \%$. Grade $3-5$ treatment-related toxicity rate was lower with pembrolizumab, $13 \%-16 \%$ vs. $35 \%$ [16].

Checkpoint blockades have shown improved treatment outcomes in the clinical trials for advanced melanoma and NSCLC patients with previous systemic treatments. Notably, the response rate of pembrolizumab was not significantly lower even in the patients with ipilimumab history $[17,18]$. Nonetheless, advanced solid tumors other than melanoma or NSCLC have not been reported to have considerable efficacy or safety of checkpoint blockades $[19,20]$. Hence, combination strategies are actively discovered to extend the target of checkpoint blockades, using chemotherapy or small molecules. $\mathrm{RT}$ is also a promising partner of checkpoint blockades in terms of the immunogenic effect as mentioned above.

\section{RT Combined with Checkpoint Blockades: Preclinical Data}

Regulatory T (Treg) cells are more radioresistant than other T cells, consequently increased by RT [21]. Naturally, an important role of Treg cells is to maintain immune tolerance, even in tumorous condition. CTLA-4 is reported to be a key target to control the suppressive function of Treg cell [22]. Thus, the combination of RT and anti-CTLA- 4 mAb has been investigated to overcome tumor immunity. Demaria et al [23] injected poorly immunogenic 4T1 metastatic mouse mammary carcinoma cells into mice, and then performed 2 by 2 arms of treatment: (RT or no-RT) $\times(C T L A-4$ mAb or control immunoglobulin $\mathrm{G}[\mathrm{lg} G])$. When RT was combined with antiCTLA-4 mAb, the survival was significantly extended and lung metastasis was controlled. In this study, CD8+ T cell was 
shown to have crucial role, while CD4+ T cell did not. Antitumor immunity was also confirmed in other types of cell lines, such as mesothelioma [24] and glioma [25].

Though the combination of RT and anti-CTLA- 4 mAb has been reported to have improved response, the unresponders are more common than the responders. In this point of view, the importance of PD-L1/PD-1 co-inhibitory pathway has been noted, which modulate microenvironment to facilitate activation of CD8+ T cells [26]. Twyman-Saint Victor et al. [27] obtained resistant murine melanoma cells from mice with relapsed tumor after the combination of RT and anti-CTLA4 $\mathrm{mAb}$. The authors found that the resistance was associated with the exhaustion of $C D 8+T$ cell and the upregulation of PD-L1 by cancer cells. After the addition of anti-PD-L1 mAb, exhausted CD8+ $T$ cell was reversed and the response was improved. In addition to effector T cells, down-regulation of tumor-infiltrating myeloid-derived suppressor cells by TNF- $\alpha$ was reported to be associated with antitumor immunity [28], and blocking of TGF- $\beta$ might be a another strategy [29]. Thus, murine melanoma cell line was a preceding model to confirm the effect of the combination of anti-PD-1/PD-L1 mAb and RT [30]. Actually, improved anti-tumor immunity has been revealed in breast [28], colon [31], and renal cell carcinoma [32].

Furthermore, impressive feature of immunotherapy, immune memory was confirmed. Zeng et al. [33] selected "cured mice," which survived more than 90 days after the combined treatment of RT and anti-PD-1 mAb for murine glioma, and injected same cell line. At the same time, naive mice were injected with same cell line. All of naive mice had $>1 \mathrm{~cm}^{3}$ tumors after 21 days of implantation, while none of cured mice had tumors after 60 days of implantation. Enhanced abscopal effect was also been reported in other study, combined treatment of RT and anti-PD-1 mAb significantly inhibited the growth of secondary unirradiated tumors, comparing with single treatment of RT or anti-PD-1 mAb [32] (Table 1).

\section{RT Combined with Checkpoint Blockades: Clinical Data}

At this time, a limited number of clinical trials reported results of combined RT and checkpoint blockades, moreover, most of them used anti-CTLA-4 mAb. A phase I/II study for patients with metastatic castration-resistant prostate cancer evaluated safety and efficacy of ipilimumab, an anti-CTLA- 4 mAb with or without radiotherapy. RT was delivered to metastatic bone lesion with 8 Gy single fraction 1 to 2 days before the first ipilimumab. The dose of ipilimumab was escalated as 3, 5 or $10 \mathrm{mg} / \mathrm{kg}$ without RT, or 3 or $10 \mathrm{mg} / \mathrm{kg}$ with RT. Response was assessed after up to 4 cycles ( 3 weeks per cycle). Among 34 patients with $10 \mathrm{mg} / \mathrm{kg}$ ipilimumab and RT, grade 3 to 4 immune-related adverse effects occurred in $18 \%$ of patients (colitis, 6\%; hepatitis, 6\%; and diarrhea, 6\%), and $\geq 50 \%$ prostate-specific antigen (PSA) decline was confirmed in $12 \%$ of patients [34]. In a phase III trial, ipilimumab was compared with placebo for metastatic castration-resistant prostate cancer patients. Single fraction RT (8 Gy) was given for metastatic bone lesion within the 2 days before the medication. I pilimumab $(10 \mathrm{mg} / \mathrm{kg})$ or placebo was administered every 3 weeks for up to 4 cycles, and continued every 3 months until disease progression or severe toxicity. A total of 799 patients were randomly assigned to ipilimumab $(n=399)$ and placebo $(n=400)$ groups. Though OS for the entire patients was not increased with ipilimumab (11.2 vs. 10.0 months, $p=0.053$ ), progression-free survival (4.0 vs. 3.1 months, $p<0.0001)$ and $\geq 50 \%$ PSA decline $(13.1 \%$ vs. $5.2 \%)$ was improved. Grade 3 to 4 immune related adverse effects were noted in 26\% of ipilimumab group and $3 \%$ of placebo group, and common immune related events were diarrhea $(15 \%$ vs. $<1 \%)$ and colitis (5\% vs. $0 \%)$. Four patients $(1 \%)$ in ipilimumab group had ipilimumab-related death [35]. These two clinical trials reported similar rate of severe immunerelated toxicity comparing with aforementioned phase III

Table 1. Checkpoint blockades and effect of radiotherapy

\begin{tabular}{cccc}
\hline Ligands on tumor cell & Receptors on T cell & Ligands on antigen-presenting cell & Checkpoint blockade \\
\hline PD-L1 and L2 & PD-1 & - & $\begin{array}{c}\text { Anti-PD-1/L1 } \\
\text { (atezolizumab, nivolumab, pembrolizumab) } \\
\text { CTLA-4 }\end{array}$ \\
& B 7 (CD80, CD86) & Anti-CTLA-4 (ipilimumab)
\end{tabular}

Effect of radiotherapy To increase antigen presentation and $C D 8+T$ cell infiltration

To stimulate tumor-specific cytotoxic T lymphocytes in the distant sites: abscopal effect

PD-L1 and L2, programmed death-ligand 1 and ligand 2; PD-1, programmed death-1; CTLA-4, cytotoxic T-lymphocyte-associated protein 4 . 
immunotherapy trials without RT, hence we can expect the combination of RT and checkpoint blockades could be a useful treatment modality. More data can be found in retrospective studies, and they may give us additional clue to identify the benefit of the combination.

In the real world, immunotherapy is usually tried for patients with systemic metastasis, thus RT may be particularly used as palliative intent. As expected, palliative RT (median dose of $30 \mathrm{~Gy}$ ) combined with ipilimumab (median dose of $10 \mathrm{mg} / \mathrm{kg}$ ) was reported to have appropriate palliative effect without significant increase of immune-related adverse effects [36]. If the abscopal effect is confirmed in clinical data, the combination of RT and immunotherapy can be more useful. Several case reports have observed the abscopal effect of local RT. A metastatic melanoma patient who had showed stable disease with ipilimumab received palliative RT (28.5 Gy in 3 fractions) when the disease progressed. Shortly after RT, there was not a response. However, a significant regression was observed in both of irradiated lesion and distant non-irradiated lesions after an additional dose of ipilimumab [37]. In another case report, a patient with metastatic lung adenocarcinoma underwent palliative RT (30 Gy in 5 fractions) concomitantly with ipilimumab. After the concomitant treatment, significant reduction of tumor size and metabolic uptake was detected in whole body, furthermore, the carcinoembryonic antigen level was normalized either [38].

Brain metastasis can be a typical model of combined treatment of RT and immunotherapy, since complete resection is difficult and systemic agents hardly penetrate the bloodbrain barrier. Survival benefit of the combination of brain RT and ipilimumab was reported in a study for brain metastatic melanoma patients, the combined treatment showed better OS (median, 21.3 vs. 4.9 months; $p=0.044$ ) than radiosurgery alone [39]. The abscopal effect observed either. A retrospective study reported treatment results of 13 patients with brain metastatic melanoma who treated with ipilimumab (3 mg/ $\mathrm{kg}$ ) and whole-brain RT (WBRT; median 30 Gy in 10 fractions) within 30 days of one another. Extracranial response rate was evaluated in 10 patients, and the response rate was 20\% (complete response 1, partial response 1, and stable disease 2). The rate of grade 3 to 4 central nervous system-related acute toxicity was low, reported in only 1 patient. Notably, all patients who had post-WBRT imaging had new or worsened intratumoral hemorrhage (median, 53 days) [40]. However, the influence of RT or ipilimumab on hemorrhage should be cautiously considered, because intratumoral hemorrhage is commonly occurred in melanoma metastases. Actually, in another study for brain metastatic melanoma patients, more intratumoral hemorrhage was occurred in brain RT alone group than brain RT and ipilimumab group (12.5\% vs. $3.9 \%$ ) [41].

In summary, clinical data for the combination of RT and checkpoint blockades are still scanty and concentrated on a certain agent, ipilimumab. Nonetheless, based on the results of several retrospective studies, we can expect that the combined treatment may be synergistic without significant increase of immune-related adverse effects.

\section{How to Combine RT with Checkpoint Blockades?}

Several technical issues should be considered for combination of immune checkpoint and RT, such as fractionation schedule and sequence of RT. We should look back on preclinical data to find appropriate fraction size. In a mouse model using B16 melanoma cells, 20 Gy was delivered in 1 fraction or 5 fractions. Increased reduction of primary tumor or distant metastasis was reported in single fractionation group rather than fractionation group. This ablative RT-initiated immunity was CD8+ T-cell dependent [42]. In other study using a fractionated schedule of RT ( 2 Gy $\times 5)$, upregulation of PDL1 was observed in murine colon carcinoma cells of mice [43]. This is the adaptive immune-evading mechanism of tumor cells, depending on IFN- $\gamma$ produced by CD8 T cell. In contrast, single fraction RT with higher dose (10 Gy) decreased the expression of PD-L1 in human prostate cancer cell lines in vitro [44].

Based on these results, ablative RT with single fraction might be suggested to initiate immunologic response better than fractionated RT. However, 2 to 5 Gy per fraction which used in aforementioned studies is a relatively conventional schedule. Fractionated RT with conventional low daily dose may be less immunologic, at least in the setting of metastatic disease. Silk et al. [41] reported that survival benefit of ipilimumab was confirmed in radiosurgery group (19.9 vs. 4.0 months; $p=0.009$ ), while not in WBRT (3.1 vs. 5.3 months; $p=$ 0.60). In this study, RT dose was 30-37.5 Gy in 10-13 fractions and 14-24 Gy in 1-5 fractions for radiosurgery. Therefore, fractionated RT with higher daily dose should be compared with single fraction RT. Actually, Dewan et al. [45] injected TSA mouse breast carcinoma or MCA38 mouse colon carcinoma cells into the both flanks of mouse. RT was given to "primary site" as 20 Gy in single fraction, 8 Gy in 3 fractions, and 6 Gy in 5 fractions. $9 \mathrm{H} 10$ monoclonal antibody against CTLA-4 was 

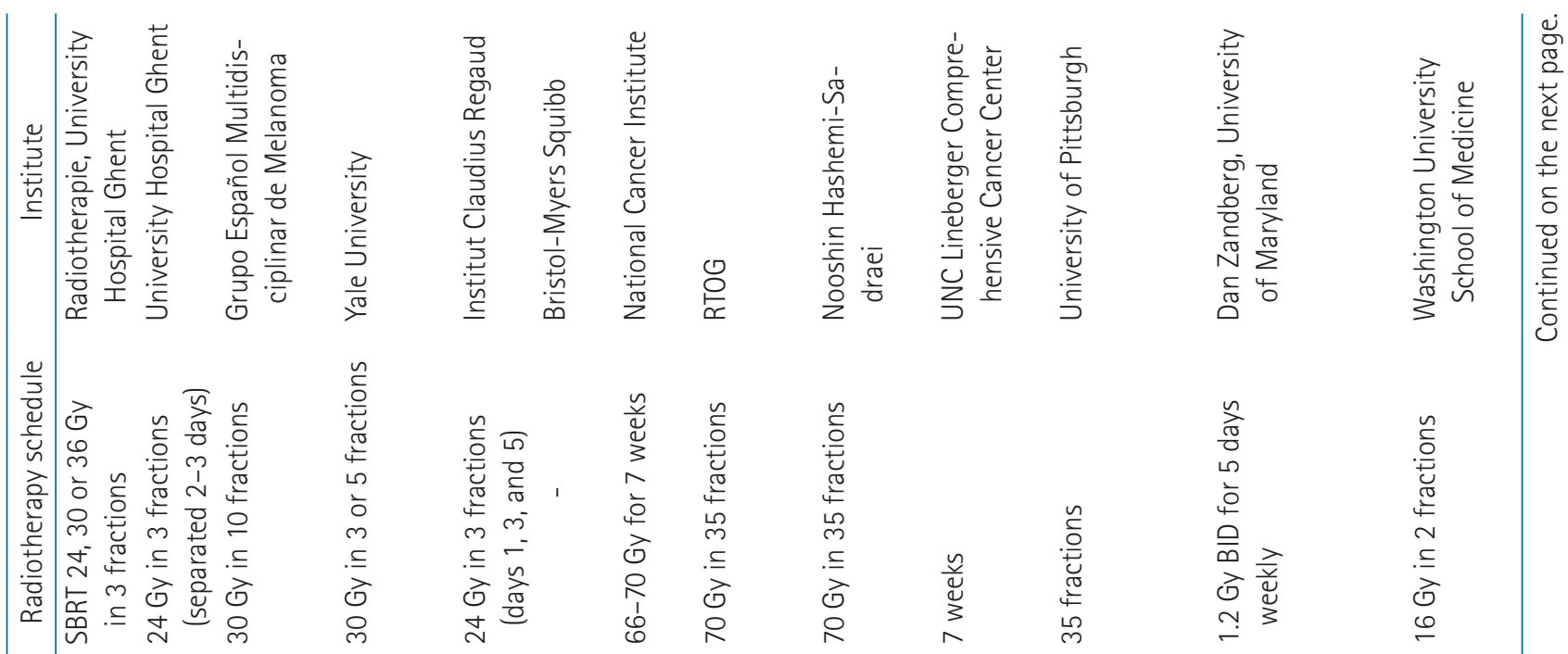

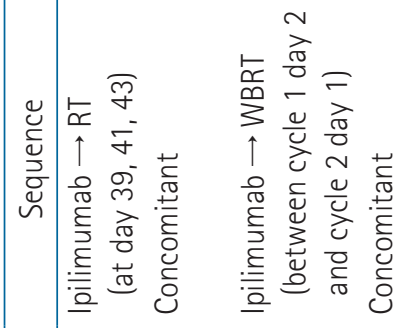

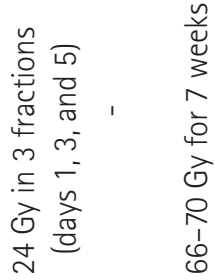

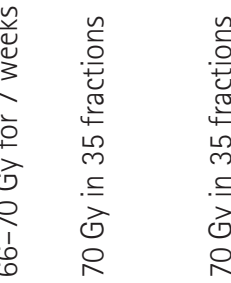

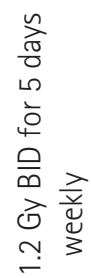

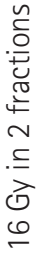
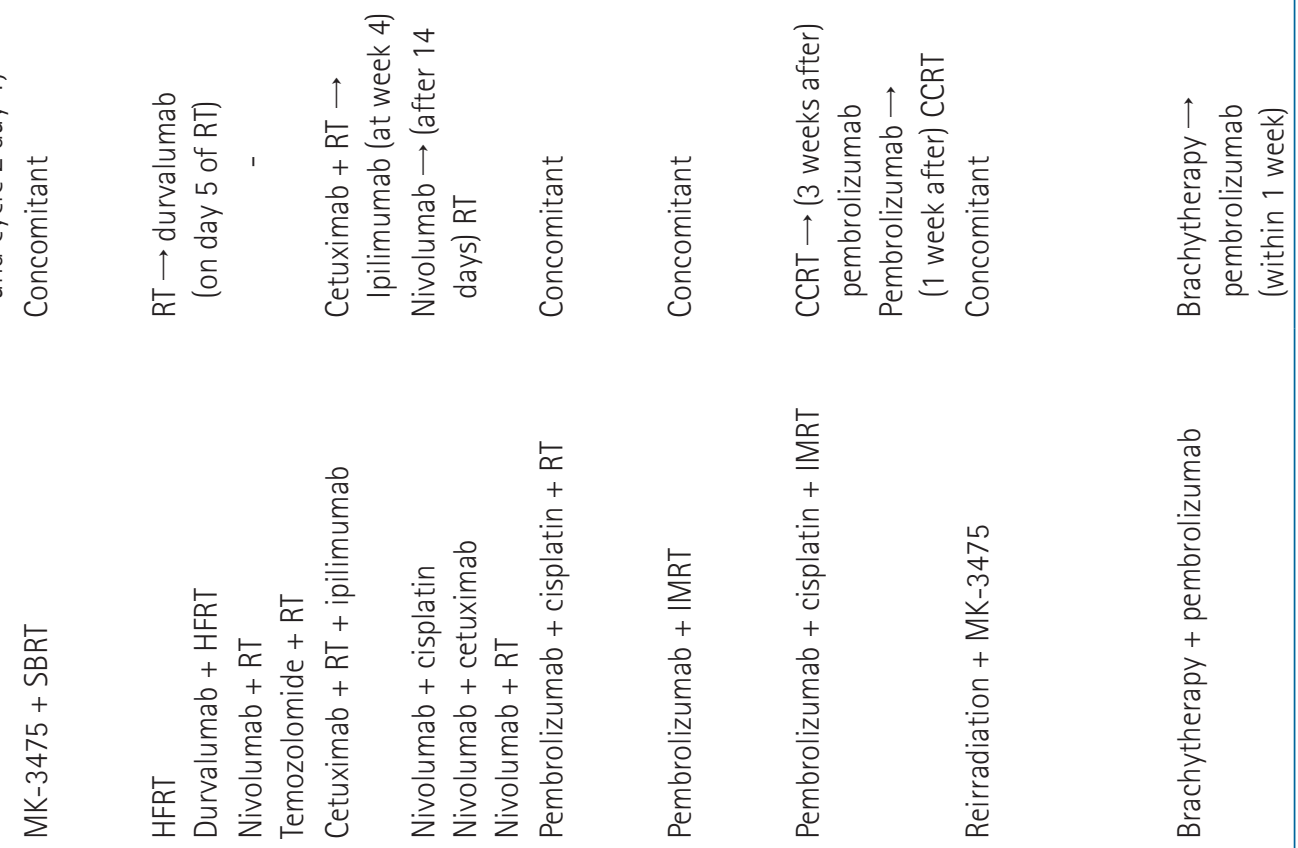

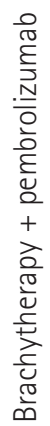

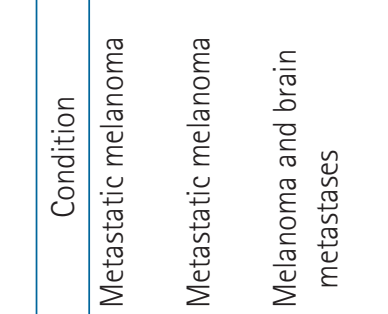

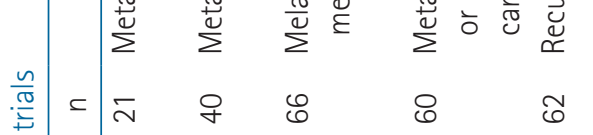

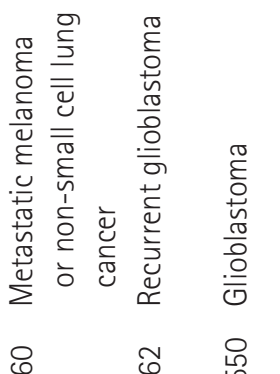

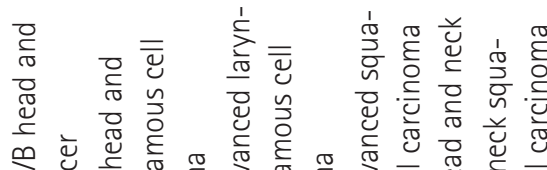

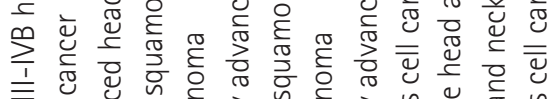

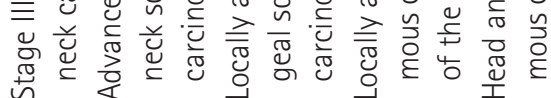

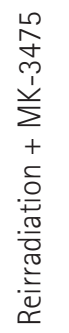

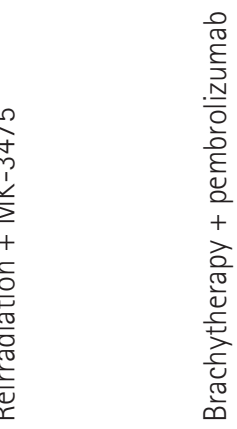

产

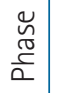

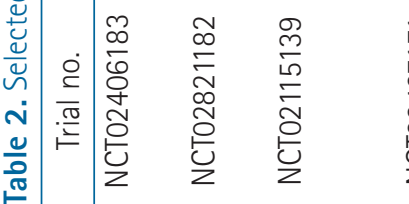

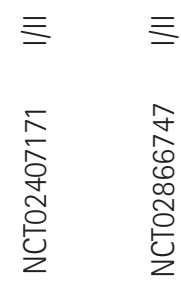

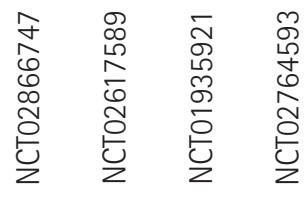

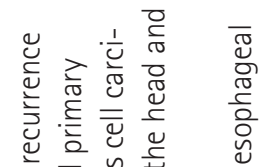
항

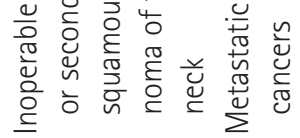

字

$\stackrel{ }{\circ}$

$\propto \stackrel{ }{\circ}$ \&

$\circ$

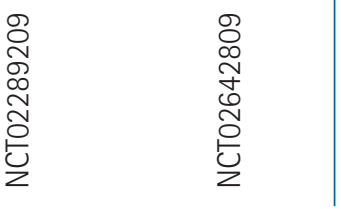




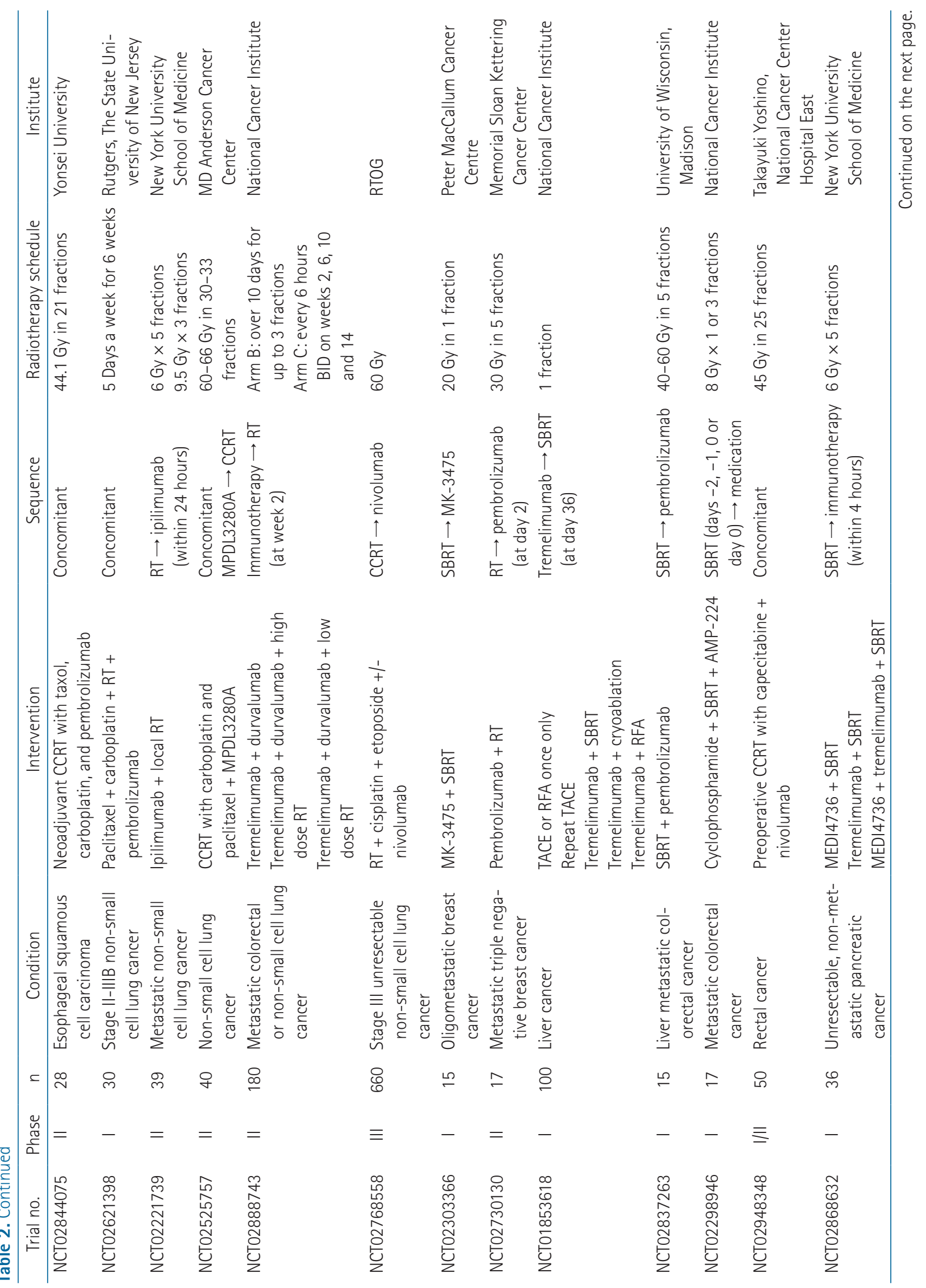




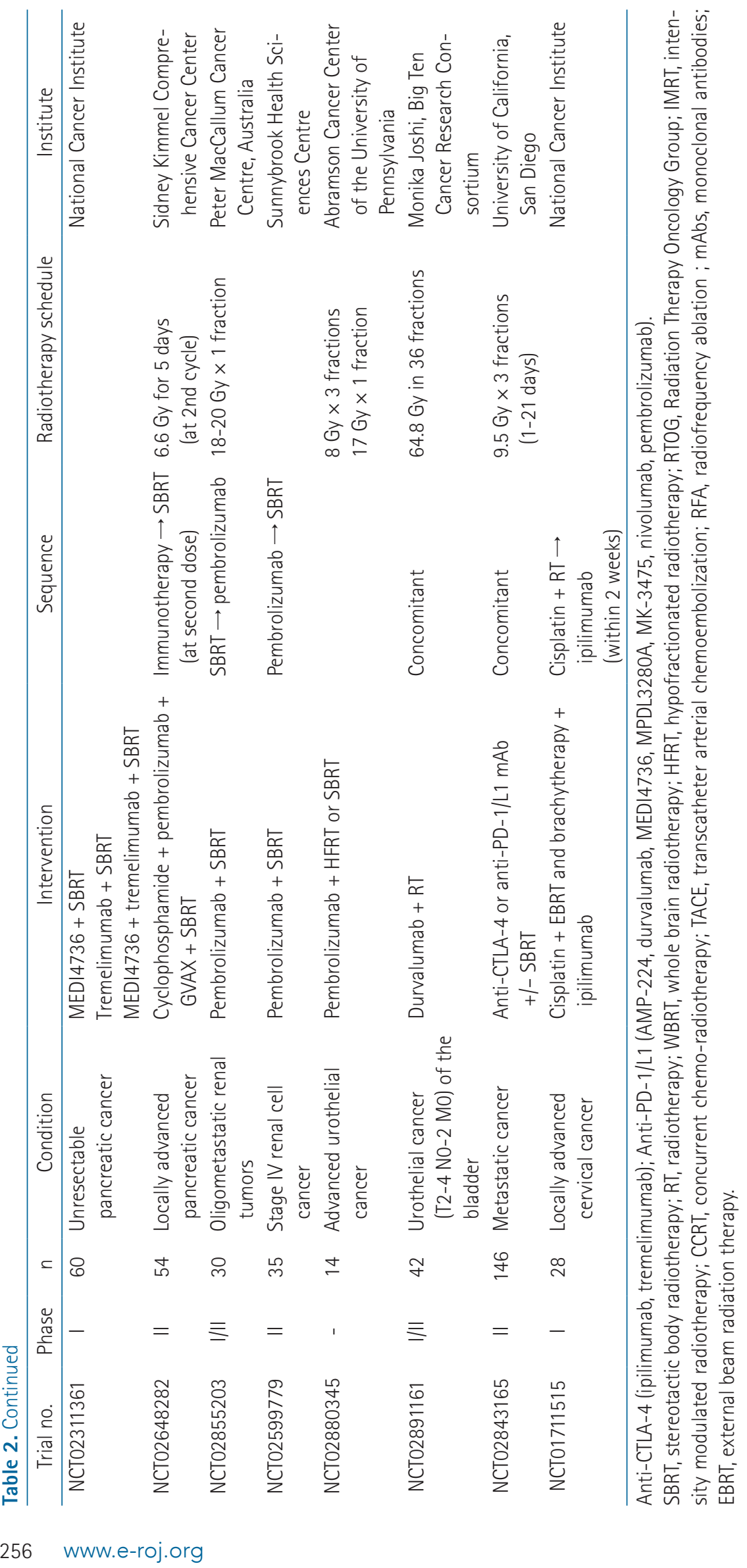

https://doi.org/10.3857/roj.2016.02033 
ROJ Radiation Oncology Journal

combination with RT or not. Interestingly, the RT regimen with $8 \mathrm{~Gy} \times 3$ fractions showed most enhanced response regardless of tumor sites.

Regarding the sequence of RT and immunotherapy, it is another uncertainty. In one study for brain metastatic melanoma, response rate was 40\% in RT after ipilimumab and $16.7 \%$ in RT before ipilimumab [41]. On the contrary, in other study for melanoma brain metastases, patients underwent radiosurgery during or before ipilimumab had better OS than those underwent radiosurgery after ipilimumab (at 1 year, 65\% vs. $56 \%$ vs. $40 \%, p=0.008$ ). However, patients underwent radiosurgery during ipilimumab showed more frequent central nervous system toxicities, for example, hemorrhage was found in $40 \%$, compared with $18 \%$ in patients underwent radiosurgery before or after ipilimumab [46]. However, small number of patients and retrospective nature is the limitations of these studies to clarify the optimal sequence of RT and immunotherapy.

\section{Ongoing Trials and Future Perspectives}

Major issues for the combined treatment of RT and checkpoint blockades can be summarized as follows. Above all, more types of cancer, beyond melanoma and NSCLC, must be considered as the indication of combined treatment. Especially, several cancers with poor prognosis or limited applicable systemic agents, such as pancreas and triple negative breast cancers, were reported to be possibly immunogenic $[47,48]$, so multiple studies have been conducted for varied types of cancer (Table 2). Additionally, anti-PD-1/L1 mAb, besides anti-CTLA-4 mAb, should be verified to have synergistic effect by the combined RT and immunotherapy. Most of preclinical and clinical studies used anti-CTLA-4 mAb, especially ipilimumab, being developed earlier than other agents. Tumor tissue studies in patients receiving neoadjuvant chemo-radiotherapy reported that PD-L1 expression was increased in tumor cells, but its influence on prognosis is very conflicting $[49,50]$. Lastly, optimal combination schedule of RT and checkpoint blockades also has to be clarified, including fractionation schedule and sequence of RT and checkpoint blockades. Although RT with higher daily dose appears more immunogenic than RT with conventional daily dose in several preclinical or retrospective studies, we should take into account that the studies used $\mathrm{RT}$ in a palliative setting. For curative intent, conventionally fractionated (around 2 Gy per day) RT is still the mainstay, concerning possible normal tissue toxicity owing to usually combined systemic agents. Consequently, conventionally
Immune checkpoint blockade combined with radiotherapy

fractionated RT should be investigated in clinical trials whether has synergistic immunogenic effect, as well as the sequence of $\mathrm{RT}$ and immune checkpoint blockade. Selected ongoing trials are summarized in Table 2.

\section{Conclusion}

Immune checkpoint blockades have been emerged as promising anticancer therapy, showing enormous progression on clinical application. Pro-immune effect of RT is expected to boost efficacy of checkpoint blockades without significant increase of immune-related adverse event. Although the evidence of combined treatment is not sufficient yet preclinical data suggest a potential benefit of combined RT and checkpoint blockades. Based on ongoing clinical trials, the latitude for clinical application of RT would be extended, from palliation to radical treatment modality, in the field of immunotherapy.

\section{Conflict of Interest}

No potential conflict of interest relevant to this article was reported.

\section{Acknowledgments}

This work was supported by the grant (No. 0820010) for Cancer Control Program from the Korea Ministry of Health \& Welfare to In Ah Kim.

\section{References}

1. Formenti SC, Demaria S. Combining radiotherapy and cancer immunotherapy: a paradigm shift. J Natl Cancer Inst 2013;105:256-65.

2. Reits $E A$, Hodge JW, Herberts $C A$, et al. Radiation modulates the peptide repertoire, enhances MHC class I expression, and induces successful antitumor immunotherapy. J Exp Med 2006;203:1259-71.

3. Gupta A, Probst HC, Vuong V, et al. Radiotherapy promotes tumor-specific effector CD8+ T cells via dendritic cell activation. J Immunol 2012;189:558-66.

4. Lugade AA Moran JP, Gerber SA, Rose RC, Frelinger JG, Lord EM. Local radiation therapy of B16 melanoma tumors increases the generation of tumor antigen-specific effector cells that traffic to the tumor. J Immunol 2005;174:7516-23.

5. Siva S, MacManus MP, Martin RF, Martin OA. Abscopal effects of radiation therapy: a clinical review for the radiobiologist. 
Cancer Lett 2015;356:82-90.

6. Lenschow DJ, Walunas TL, Bluestone JA. CD28/B7 system of T cell costimulation. Annu Rev Immunol 1996;14:233-58.

7. Sansom DM. CD28, CTLA-4 and their ligands: who does what and to whom? Immunology 2000;101:169-77.

8. Keir ME, Butte MJ, Freeman GJ, Sharpe AH. PD-1 and its ligands in tolerance and immunity. Annu Rev Immunol 2008;26:677-704.

9. Hodi FS, O'Day SJ, McDermott DF, et al. Improved survival with ipilimumab in patients with metastatic melanoma. N Engl J Med 2010;363:711-23.

10. Robert C, Thomas L, Bondarenko I, et al. Ipilimumab plus dacarbazine for previously untreated metastatic melanoma. N Engl J Med 2011;364:2517-26.

11. Maio M, Grob JJ, Aamdal S, et al. Five-year survival rates for treatment-naive patients with advanced melanoma who received ipilimumab plus dacarbazine in a phase III trial. J Clin Oncol 2015;33:1191-6.

12. Larkin J, Chiarion-Sileni V, Gonzalez R, et al. Combined nivolumab and ipilimumab or monotherapy in untreated melanoma. N Engl J Med 2015;373:23-34.

13. Robert C, Schachter J, Long GV, et al. Pembrolizumab versus ipilimumab in advanced melanoma. N Engl J Med 2015;372:2521-32.

14. Brahmer J, Reckamp KL, Baas $P$, et al. Nivolumab versus docetaxel in advanced squamous-cell non-small-cell lung cancer. N Engl J Med 2015;373:123-35.

15. Borghaei $H$, Paz-Ares $L$, Horn $L$, et al. Nivolumab versus docetaxel in advanced nonsquamous non-small-cell lung cancer. N Engl J Med 2015;373:1627-39.

16. Herbst RS, Baas P, Kim DW, et al. Pembrolizumab versus docetaxel for previously treated, PD-L1-positive, advanced non-small-cell lung cancer (KEYNOTE-010): a randomised controlled trial. Lancet 2016;387:1540-50.

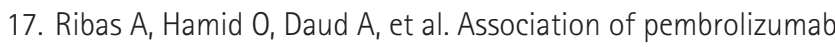
with tumor response and survival among patients with advanced melanoma. JAMA 2016;315:1600-9.

18. Ribas A, Puzanov I, Dummer R, et al. Pembrolizumab versus investigator-choice chemotherapy for ipilimumab-refractory melanoma (KEYNOTE-002): a randomised, controlled, phase 2 trial. Lancet Oncol 2015;16:908-18.

19. Patnaik A, Kang SP, Rasco D, et al. Phase I study of pembrolizumab (MK-3475; Anti-PD-1 monoclonal antibody) in patients with advanced solid tumors. Clin Cancer Res 2015;21:4286-93.

20. Royal RE, Levy $C_{1}$ Turner $K_{1}$ et al. Phase 2 trial of single agent Ipilimumab (anti-CTLA-4) for locally advanced or metastatic pancreatic adenocarcinoma. J Immunother 2010;33:828-33.

21. Kachikwu EL, Iwamoto KS, Liao YP, et al. Radiation enhances regulatory $T$ cell representation. Int J Radiat Oncol Biol Phys 2011;81:1128-35.
22. Wing $K$, Onishi $Y$, Prieto-Martin $P$, et al. CTLA-4 control over Foxp3+ regulatory $T$ cell function. Science 2008;322:271-5.

23. Demaria S, Kawashima N, Yang AM, et al. Immune-mediated inhibition of metastases after treatment with local radiation and CTLA-4 blockade in a mouse model of breast cancer. Clin Cancer Res 2005;11(2 Pt 1):728-34.

24. Wu L, Wu MO, De la Maza $L$, et al. Targeting the inhibitory receptor CTLA-4 on T cells increased abscopal effects in murine mesothelioma model. Oncotarget 2015;6:12468-80.

25. Yoshimoto $Y$, Suzuki Y, Mimura K, et al. Radiotherapy-induced anti-tumor immunity contributes to the therapeutic efficacy of irradiation and can be augmented by CTLA-4 blockade in a mouse model. PLoS One 2014;9:e92572.

26. Liang $H$, Deng $L$, Chmura $S$, et al. Radiation-induced equilibrium is a balance between tumor cell proliferation and T cell-mediated killing. J Immunol 2013;190:5874-81.

27. Twyman-Saint Victor $C_{1}$ Rech AJ, Maity $A$, et al. Radiation and dual checkpoint blockade activate non-redundant immune mechanisms in cancer. Nature 2015;520:373-7.

28. Deng L, Liang $H$, Burnette $B$, et al. Irradiation and anti-PD-L1 treatment synergistically promote antitumor immunity in mice. J Clin Invest 2014;124:687-95.

29. Vanpouille-Box C, Diamond JM, Pilones KA, et al. TGF $\beta$ is a master regulator of radiation therapy-induced antitumor immunity. Cancer Res 2015;75:2232-42.

30. Pilon-Thomas S, Mackay A, Vohra N, Mule JJ. Blockade of programmed death ligand 1 enhances the therapeutic efficacy of combination immunotherapy against melanoma. J Immunol 2010;184:3442-9.

31. Sharabi $A B$, Nirschl $C J$, Kochel $C M$, et al. Stereotactic radiation therapy augments antigen-specific PD-1-mediated antitumor immune responses via cross-presentation of tumor antigen. Cancer Immunol Res 2015;3:345-55.

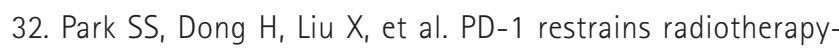
induced abscopal effect. Cancer Immunol Res 2015;3:610-9.

33. Zeng J, See AP, Phallen J, et al. Anti-PD-1 blockade and stereotactic radiation produce long-term survival in mice with intracranial gliomas. Int J Radiat Oncol Biol Phys 2013;86:343-9.

34. Slovin SF, Higano CS, Hamid O, et al. Ipilimumab alone or in combination with radiotherapy in metastatic castrationresistant prostate cancer: results from an open-label, multicenter phase I/II study. Ann Oncol. 2013;24:1813-21.

35. Kwon ED, Drake CG, Scher HI, et al. I pilimumab versus placebo after radiotherapy in patients with metastatic castrationresistant prostate cancer that had progressed after docetaxe chemotherapy (CA184-043): a multicentre, randomised, double-blind, phase 3 trial. Lancet Oncol 2014;15:700-12.

36. Barker CA, Postow MA, Khan SA, et al. Concurrent radiotherapy and ipilimumab immunotherapy for patients with melanoma. Cancer Immunol Res 2013;1:92-8. 
37. Postow MA, Callahan MK, Barker CA, et al. Immunologic correlates of the abscopal effect in a patient with melanoma. N Engl J Med 2012;366:925-31.

38. Golden EB, Demaria S, Schiff PB, Chachoua A, Formenti SC. An abscopal response to radiation and ipilimumab in a patient with metastatic non-small cell lung cancer. Cancer Immunol Res 2013;1:365-72.

39. Knisely JP, Yu JB, Flanigan J, Sznol M, Kluger HM, Chiang VL. Radiosurgery for melanoma brain metastases in the ipilimumab era and the possibility of longer survival. J Neurosurg 2012;117:227-33.

40. Gerber NK, Young RJ, Barker CA, et al. Ipilimumab and whole brain radiation therapy for melanoma brain metastases. J Neurooncol 2015;121:159-65.

41. Silk AW, Bassetti MF, West BT, Tsien Cl, Lao CD. Ipilimumab and radiation therapy for melanoma brain metastases. Cancer Med 2013;2:899-906.

42. Lee $Y$, Auh $S L$, Wang $Y$, et al. Therapeutic effects of ablative radiation on local tumor require $\mathrm{CD} 8+\mathrm{T}$ cells: changing strategies for cancer treatment. Blood 2009;114:589-95.

43. Dovedi SJ, Adlard AL, Lipowska-Bhalla G, et al. Acquired resistance to fractionated radiotherapy can be overcome by concurrent PD-L1 blockade. Cancer Res 2014;74:5458-68.

44. Bernstein MB, Garnett $C T$, Zhang $H$, et al. Radiation-induced modulation of costimulatory and coinhibitory T-cell signaling molecules on human prostate carcinoma cells promotes productive antitumor immune interactions. Cancer Biother Radiopharm 2014;29:153-61.

45. Dewan MZ, Galloway AE, Kawashima N, et al. Fractionated but not single-dose radiotherapy induces an immune-mediated abscopal effect when combined with anti-CTLA-4 antibody. Clin Cancer Res 2009;15:5379-88.

46. Kiess AP, Wolchok JD, Barker CA, et al. Stereotactic radiosurgery for melanoma brain metastases in patients receiving ipilimumab: safety profile and efficacy of combined treatment. Int J Radiat Oncol Biol Phys 2015;92:368-75.

47. Lutz ER, Wu AA, Bigelow $E$, et al. Immunotherapy converts nonimmunogenic pancreatic tumors into immunogenic foc of immune regulation. Cancer Immunol Res 2014;2:616-31.

48. Stagg J, Allard B. Immunotherapeutic approaches in triplenegative breast cancer: latest research and clinical prospects. Ther Adv Med Oncol 2013;5:169-81.

49. Lim SH, Hong M, Ahn S, et al. Changes in tumour expression of programmed death-ligand 1 after neoadjuvant concurrent chemoradiotherapy in patients with squamous oesophageal cancer. Eur J Cancer 2016;52:1-9.

50. Hecht M, Buttner-Herold M, Erlenbach-Wunsch $K_{1}$ et al. PD-L1 is upregulated by radiochemotherapy in rectal adenocarcinoma patients and associated with a favourable prognosis. Eur J Cancer 2016;65:52-60. 